\title{
Potential Therapeutic Modalities of Reawakening Fetal Hemoglobin Simulated by Reaction Systems
}

\author{
Mani Mehraei ${ }^{1}$, Benedek Nagy ${ }^{1}$, Nımet Ilke Akcay ${ }^{2}$, \\ Şükrü Tüzmen ${ }^{3}$
}

${ }^{1}$ Department of Applied Mathematics and Computer Science,

Eastern Mediterranean University, Famagusta, North Cyprus, Mersin-10, Turkey, mani.mehraei@emu.edu.tr, benedek.nagy@emu.edu.tr

${ }^{2}$ Faculty of Medicine, Eastern Mediterranean University,

Famagusta, North Cyprus, Mersin-10, Turkey, ilke.cetin@emu.edu.tr

${ }^{3}$ Department of Biological Sciences, Eastern Mediterranean University, Famagusta, North Cyprus, Mersin-10, Turkey, sukru.tuzmen@emu.edu.tr

\begin{abstract}
Thalassemia syndromes are a diverse group of inherited genetic disorders. There are different types of thalassemia disorders, such as, $\beta$-thalassemia, which is also called Mediterranean anemia, that is an inherited disease that played a major role in the American thriller movie, "Dying of the light" starring Nicolas Cage (Dec. 2014). In this study, we focus on the beta-globin ( $\beta$-globin) gene family related disorders. We seek potential amelioration strategies for $\beta$-thalassemia and sickle cell anemia via $\gamma$-globin gene induction. In this work, a simulation model is developed, utilizing a reaction systems methodology. These systems are finite and based on a discrete time scale and can be used to describe and analyze complex biological systems and biological phenomenon. In our model, simulations of normal and abnormal cases of fetal, to adult hemoglobin switching developmental stage are illustrated. Various types of known and potential treatment strategies for $\beta$-thalassemia and sickle cell anemia cases from the literature have been utilized to validate our model, used for identifying new potential treatments to be tested by molecular biologists, in the future studies. Moreover, we propose a novel potential simulation, as a therapeutic means, for $\beta$-thalassemia and sickle cell anemia, by identifying FOG1 as a potential target. Finally, our proposed model, based on a reaction systems methodology, shows that inhibition of FOGl expression by using methods, such as, RNAi induces $\gamma$-globin gene expression and can compensate for the lack of beta-globin in patients suffering from $\beta$-globin gene related diseases, such as, $\beta$-thalassemia and sickle cell anemia.
\end{abstract}

Keywords: bioinformatics; hemoglobin switching; beta-globin; beta-thalassemia; reaction systems; simulation; modeling biological systems 


\section{Introduction}

Diseases related to the hemoglobin $(\mathrm{Hb})$ protein can result from either structural aberrations, that give rise to "hemoglobinopathies", or defects in the synthesis of one or more of the polypeptide chains of $\mathrm{Hb}$, which lead to thalassemias [17]. The thalassemia syndromes are a diverse group of inherited disorders that can be characterized according to their insufficient synthesis or absent production of one or more of the globin chains. Here we will emphasize the beta-globin ( $\beta$-globin) gene family related disorders, especially the potential amelioration strategies for $\beta$-thalassemias via $\gamma$-globin gene upregulation. To date more than 200 mutations are reported causing various levels of $\beta$-globin gene defects [17], which are known to produce $\beta$-thalassemia. $\beta$-thalassemia and sickle-cell anemia diseases are caused by mutation(s) in $\beta$-globin gene, which result in defective adult Hemoglobin ( $\mathrm{HbA}$ ) and lead to various abnormal phenotypes. $\beta$-thalassemia itself is a common blood disorder worldwide, especially endemic in regions such as Mediterranean basin, Middle Eastern countries, Central and South Eastern Asia, Northern part of Africa, and India. Every year, thousands of infants worldwide are born with this disease.

$\beta$-thalassemia is an inherited blood disorder and the global annual incident rate has been reported to be 1 in 100,000 live births [8]. Thus, preventing it by educating and informing people, and introducing novel treatment strategies is essential. Hemoglobin Switching refers to a developmental stage of globin gene regulation (Fig. 1). In the case of the fetal to adult globin gene expression switch, $\gamma$-globin gene expression is up-regulated during the first six months of gestational age before birth, and then starts going down, continuing after birth. This gradual down-regulation of $\beta$-globin gene is compensated by the gradual up-regulation of $\gamma$-globin gene expression starting from just before the third month of gestational age before birth, and continuing to be up-regulated after birth replacing $\gamma$-globin gene production in a healthy human being. Overall, this phenomenon results in down regulation of fetal hemoglobin $(\mathrm{HbF})$ and up regulation of adult hemoglobin $(\mathrm{HbA})$ in a healthy adult developmental stage (Fig. 1). In case of any defect in $\beta$ globin gene, this picture is presented with a lack of or no production of $\mathrm{HbA}$. These defects should be supported/corrected by means of treating the individuals with these syndromes, utilizing blood transfusions and certain drug treatments. Therefore, one of the strategies in recent decades was related to compensating the lack or loss of $\mathrm{HbA}$ by inducing $\gamma$-globin gene production, which would result in up-regulation of Fetal Hemoglobin $(\mathrm{HbF})$ molecule in turn replacing the lost function of the $\mathrm{HbA}[2]$.

Facilitation and inhibition are two fundamental mechanisms for functioning biochemical reactions [6]. Reaction Systems are formal models that work on a discrete timescale in a parallel manner to investigate and analyze biochemical reactions and the interaction among them [7]. A formal reaction has been considered as a triplet $a=(\mathrm{R}, \mathrm{I}, \mathrm{P})$, where $\mathrm{R}$ is the set of reactants, $\mathrm{I}$ is the set of 
inhibitors, and $\mathrm{P}$ is the set of products [6]. A reaction is enabled if all elements of set $\mathrm{R}$, are present and all elements of set I are absent in the actual "state" of the system. If a reaction goes, then the result will be set $\mathrm{P}$, as the product that is set $\mathrm{R}$ produced the elements of set $\mathrm{P}$. Otherwise a reaction is not enabled and the product of such reaction is the empty set. For example, if we define reactions $a_{1}=(\{\mathrm{A}, \mathrm{B}\},\{\mathrm{C}\},\{\mathrm{A}, \mathrm{D}\}), a_{2}=(\{\mathrm{E}\},\{\mathrm{A}\},\{\mathrm{A}, \mathrm{B}\})$, and initial state to be $\{\mathrm{A}, \mathrm{B}, \mathrm{E}\}$, then reaction $a_{1}$ is enabled since both reactants $\mathrm{A}$ and $\mathrm{B}$ are available and there is no $\mathrm{C}$ present to inhibits the reaction. Thus, the products of reaction $a_{2}$ are produced which are A and D. On the other hand, $a_{2}$ is not enabled since A as an inhibitor is available and does not let this reaction to go. Therefore, the result will be union of $\{A, D\}$ and empty set which is the set of products $\{A, D\}$.

In a biological system, there are phenomena that can easily be represented in a binary way, e.g., a gene can be down regulated or upregulated, a complex could be formed or being in parts. Therefore, we believe that relatively simple models as Reaction Systems are opt to model some biological phenomena. In this paper, we have exploited a Reaction System protocol to simulate hemoglobin switching process in case, where a genetic defect occurs in $\beta$-globin gene. This enabled us to validate qPCR data of known and experimental drugs to show the efficacy of these strategies. Ultimately, by considering a novel initial state, we came up with new strategy, which can be used to increase $\gamma$-globin gene induction.

\section{Biological Context (Targets for Various Potential Therapeutic Models)}

In a healthy human adult, c-Myb as a member of MYB family activates transcription of KLF1 gene, which is a transcription factor needed for $\gamma$-globin gene expression. KLF1 binds to promoter of BCL11A gene and activates the transcription of BCL11A gene [13]. BCL11A protein binds to NuRD complex, which contains HDAC1/2, CHD3/4, and MBD2 [10, 17]. BCL11A together with NuRD complex physically interact with SOX6 and has molecular interaction with FOG1 and GATA1 transcription factors [18]. These transcription factor complexes are also essential for $\gamma$-globin gene expression. Finally, this multiprotein complex which includes NuRD complex, BCL11A, and Erythroid Transcription Factors, SOX6, FOG1, and GATA1 [3] inhibits $\gamma$-globin gene expression [17]. This process is illustrated in Fig. 2. 


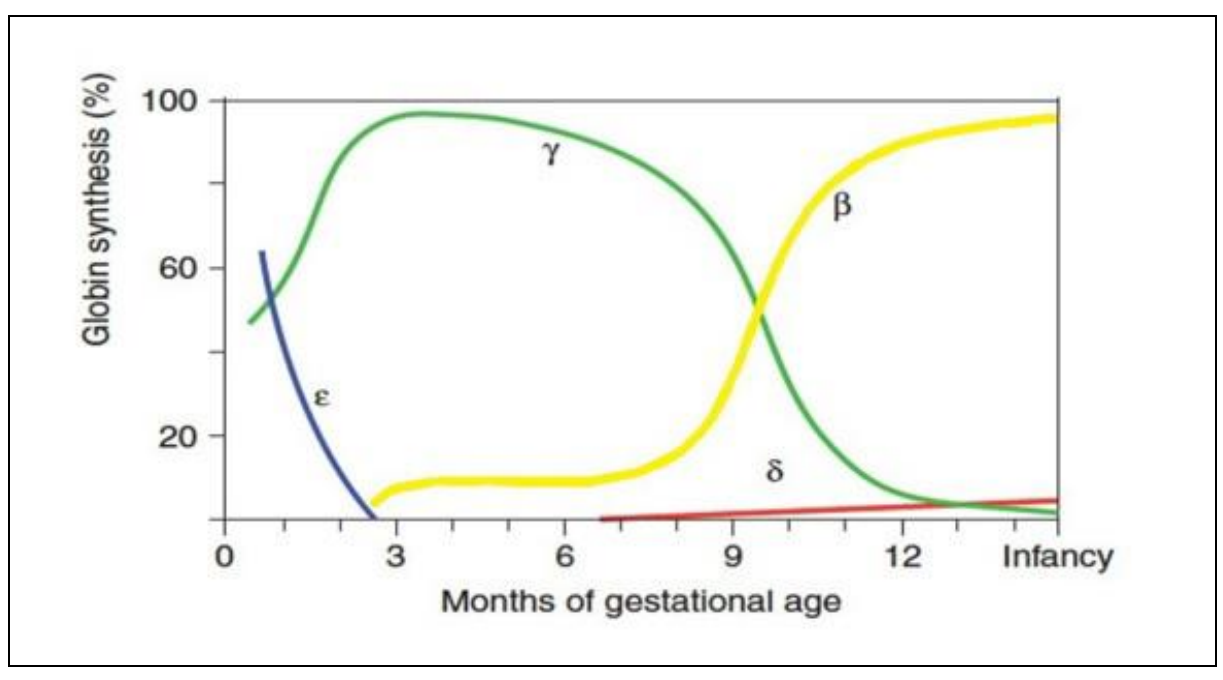

Figure 1

Fetal to adult hemoglobin switching (Adapted from [17])

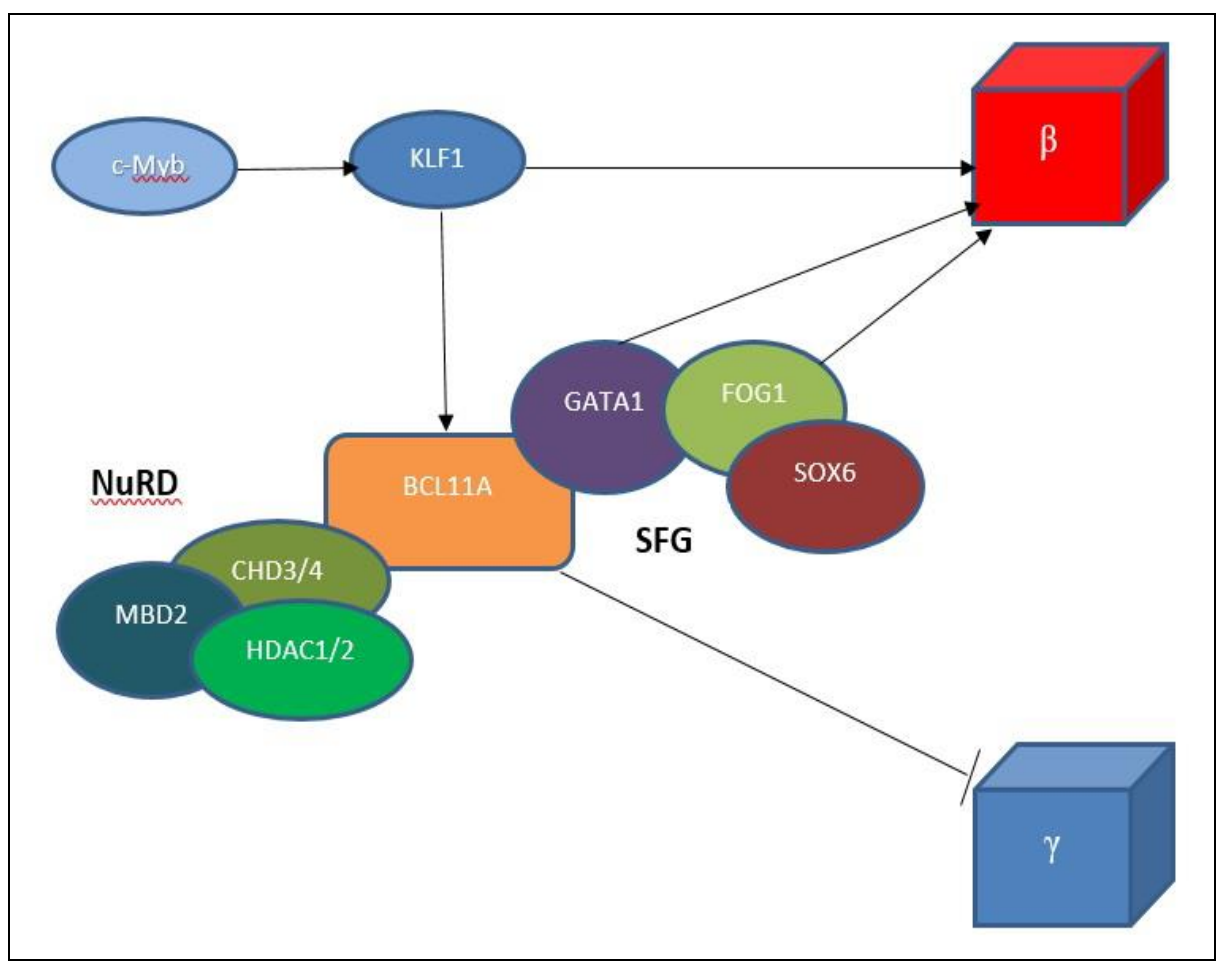

Figure 2

Hemoglobin switching pathway (Adapted from [3]) 


\section{Exploiting Reaction Systems to Model Hemoglobin Switching Process}

As mentioned earlier, a reaction can be shown as a triplet $a=(R, I, P)$, where $R$ is the set of reactants, $I$ set of inhibitors, and $P$ is the set of products [6]. A reaction system is a pair $(O, A)$ of sets of objects and reactions. To model our system in term of reaction systems we are considering these objects as possible elements of the set $O=\{C, K, B, H, N, F, G, S, S F G, \beta, \gamma\}$. Where objects corresponding to these symbols are illustrated in Table 1 .

If one, or some of these objects appear to be in a state or in reactions, it means those certain gene expressions are upregulated, otherwise disappearing or nonexistence means down regulation. We have not considered other elements of $\mathrm{NuRD}$ complex to emphasize mostly on the role of HDAC1/2 in $\gamma$-globin gene induction. Furthermore, SOX6 along with GATA1 and FOG1 are called erythroid transcription factors (SFG) which are interacting with BCL11A $[3,19]$.

The reactions, i.e., the elements of $\mathrm{A}$ in our system:

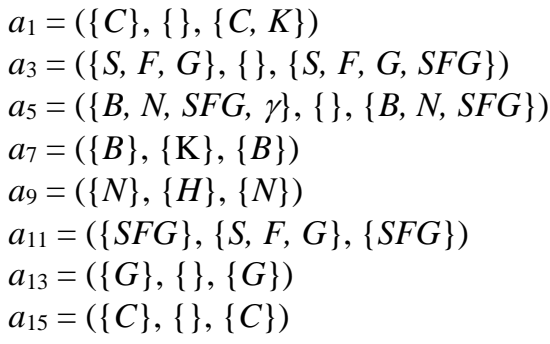

$$
\begin{aligned}
& a_{2}=(\{C, K\},\{\},\{C, K, B\}) \\
& a_{4}=(\{H\},\{\},\{H, N\}) \\
& a_{6}=(\{K, G, F\},\{\},\{K, G, F, \beta\}) \\
& a_{8}=(\{\},\{B\},\{\gamma\}) \\
& a_{10}=(\{\},\{N\},\{\gamma\}) \\
& a_{12}=(\{\},\{S F G\},\{\gamma\}) \\
& a_{14}=(\{F\},\{\},\{F\}) \\
& a_{16}=(\{S\},\{\},\{S\})
\end{aligned}
$$

Reaction $a_{1}$ illustrates that up regulation of C-Myb results into up regulation of KLF1. Reaction $a_{2}$ shows that up regulation of C-Myb and KLF1 leads to up regulation of BCL11A. Then, $a_{3}$ illustrates that up regulation of SOX6, FOG1, and GATA1 indicates up regulation of these erythroid transcription factors as a complex; and $a_{4}$ shows that up regulation of HDAC1/2 leads to up regulation of NuRD complex. We have not considered other components of NuRD complex in this system. Reaction $a_{5}$ indicates that binding of BCL11A with NuRD complex and erythroid transcription factors GATA1, FOG1, and SOX6 leads to inhibition of $\gamma$-globin gene expression. Further, $a_{6}$ shows that KLF1, GATA1, and FOG1 are transcription factors of $\beta$-globin gene. Then $a_{7}$ mentions that down regulation of KLF1 leads to down regulation of BCL11A. The reactions $a_{8}, a_{10}$, and $a_{12}$ indicate that down regulation of either BCL11A, NuRD complex, or erythroid transcription factors GATA1, FOG1, and SOX6 leads to $\gamma$-globin gene induction; while $a_{9}$ shows that down regulation of HDAC1/2 leads to down regulation of NuRD complex. Further, $a_{11}$ indicates that down regulation of either GATA1, FOG1, or SOX6 leads to down regulation of these erythroid transcription factors as a complex. Finally, $a_{13}, a_{14}, a_{15}$, and $a_{16}$ indicate that if GATA1, FOG1, C-MYB, or SOX6 are upregulating, they will remain to be upregulated. 
Table 1

Descriptions related to objects in set $O$

\begin{tabular}{|c|l|}
\hline Object's symbol & Object \\
\hline$C$ & C-Myb \\
$K$ & KLF1 \\
$B$ & BCL11A \\
$H$ & HDAC1/2 \\
$N$ & NuRD complex \\
$F$ & FOG1 \\
$G$ & GATA1 \\
$S$ & SOX6 \\
$S F G$ & Erythroid Transcription Factors SOX6, FOG1, and \\
$\beta$ & GATA1 \\
$\gamma$ & Beta globin \\
\hline
\end{tabular}

The reaction system $H_{0}=(O, A)$ is able to describe (simulate) the hemoglobin switching process of a healthy person. We considered initial state for the normal case of fetal hemoglobin switching to be $\{C, H, G, F, S, \gamma\}$. Then reactions indexed by $1,3,4,8,10,12,13,14,15$, and 16 are allowed. After the first round, new state $\{C, K, H, N, G, F, S, S F G, \gamma\}$ is obtained. Then reactions with indices 1 , $2,3,4,6,8,13,14,15,16$ are allowed. After the second round, new state is $\{C, K$, $B, H, N, G, F, S, S F G, \beta, \gamma\}$. Then reactions with numbers $1,2,3,4,5,6,13,14$, 15 , and 16 are enabled. After third round, the new state of the system is $\{C, K, B$, $H, N, G, F, S, S F G, \beta$. This is the last state which is a fix point of the system and it does not change anymore. That is $\beta$ has started to be up regulated while $\gamma$ is down regulating. The result of the simulation is the expected outcome for the healthy case.

\section{Extended Reaction System to Deal with Disease and Treatment Cases}

To extend Reaction System to cover cases related to $\beta$-thalassemia and treatment options, new objects are added and reactions are edited to the previous ones: $O^{\prime}=\{I, D, M\}$, where objects corresponding to these symbols are illustrated on Table 2 . 
Table 2

Descriptions related to objects in set $O$,

\begin{tabular}{|c|l|}
\hline Object's symbol & Object \\
\hline$I$ & BCL11A down regulator \\
$D$ & KLF1 deactivator \\
$M$ & Beta globin gene mutation \\
\hline
\end{tabular}

The reactions of our extended reaction system are as follows:

$$
\begin{array}{ll}
a_{1}^{\prime}=(\{C\},\{D\},\{C, K\}) & a_{2}^{\prime}=(\{C, K\},\{I\},\{C, K, B\}) \\
a_{3}^{\prime}=(\{S, F, G\},\{\},\{S, F, G, S F G\}) & a_{{ }^{\prime}}=(\{H\},\{\},\{H, N\}) \\
a^{\prime}=(\{B, N, S F G, \gamma\},\{\},\{B, N, S F G\}) & a^{\prime}{ }_{6}=(\{K, G, F\},\{M\},\{K, G, F, \beta\}) \\
a^{\prime}=(\{B\},\{K\},\{B\}) & a^{\prime}{ }_{8}=(\{\},\{B\},\{\gamma\}) \\
a_{9}^{\prime}=(\{N\},\{H\},\{N\}) & a_{10}^{\prime}=(\{\},\{N\},\{\gamma\}) \\
a_{11}^{\prime}=(\{S F G\},\{S, F, G\},\{S F G\}) & a_{12}^{\prime}=(\{\},\{S F G\},\{\gamma\}) \\
a_{13}^{\prime}=(\{G\},\{\},\{G\}) & a_{14}^{\prime}=(\{F\},\{\},\{F\}) \\
a_{15}^{\prime}=(\{M\},\{\},\{M\}) & a_{16}^{\prime}=(\{C\},\{\},\{C\}) \\
a_{17}^{\prime}=(\{D\},\{\},\{D\}) & a_{18}^{\prime}=(\{I\},\{\},\{I\}) \\
a^{\prime}{ }_{19}=(\{S\},\{\},\{S\}) &
\end{array}
$$

In reaction $a_{1}^{\prime}, D$ stands for KLF1 deactivator. When $D$ is presents, it doesn't let KLF1 to be transcribed so that its level decreases. In $a{ }_{2}$, I plays the role of BCL11A down regulator. Thus, whenever $I$ is present, KLF1 cannot activate transcription of BCL11A. In $a_{6}$, when mutation has happened in $\beta$-globin gene, transcription factors of $\beta$ are not able to transcribe this DNA to mRNA anymore, so that $\beta$-globin gene expression decreases. $a^{\prime}{ }_{15}, a^{\prime}{ }_{17}$, and $a^{\prime}{ }_{18}$ show that if mutation has happened, KFL1 deactivator exist, or BCL11A down regulator is present, they remain present.

The reaction system $H=\left(O \cup O^{\prime},\left\{a_{1}^{\prime}, a_{2}^{\prime}, \ldots, a_{19}^{\prime}\right\}\right)$ is able to describe (simulate) the hemoglobin switching process also in a patient who has $\beta$-globin gene mutation.

\section{Simulation Results}

In this section, simulation results in a severe $\beta$-thalassemia patient with possible treatments found in the literature, are illustrated. Moreover, potential therapeutic modalities are proposed by referring to Reaction System simulation results. For obtaining the simulation results, we have written a $\mathrm{C}++$ program on a personal computer. 


\subsection{Simulation of $\beta$-globin Gene Mutations}

To simulate the case when mutations occur in $\beta$-globin gene, we have considered initial state to be $\{C, H, G, F, S, \gamma, M\}$. Then reactions with indices $1,3,4,8,10$, $12,13,14,15,16$, and 19 are allowed. Thus, after these reactions take place, after the first round, the new state $\{C, K, H, N, G, F, S, S F G, \gamma, M\}$ is reached. In this state reactions indexed by $1,2,3,4,8,13,14,15,16$ and 19 are allowed. After second round, the new state is $\{C, K, B, H, N, G, F, S, S F G, \gamma, M\}$. Then reactions indexed by $1,2,3,4,5,7,13,14,15,16$ and 19 are enabled. Then the last state which is the fix point of the system and does not change anymore is $\{C, K, B, H$, $N, G, F, S, S F G, M$. Thus, in case of mutation in $\beta$-globin gene, expression of $\beta$ globin gene is down regulated. Moreover, $\gamma$-globin gene expression is down regulated after fetal to adult hemoglobin switching. The steps are illustrated in Fig. 3. As one can observe the simulation gives exactly the expected result also for the ill case.

$\{C, H, G, F, S, \gamma, M\}$

Where the allowed reactions have the following indices:

$1,3,4,8,10,12,13,14,15,16,19$

$\{C, K, H, N, G, F, S, S F G, \gamma, M\}$

Where the allowed reactions have the following indices:

$1,2,3,4,8,13,14,15,16,19$

$\{C, K, B, H, N, G, F, S, S F G, \gamma, M\}$

Where the allowed reactions have the following indices:

$1,2,3,4,5,7,13,14,15,16,19$

$\{C, K, B, H, N, G, F, S, S F G, M\}$

Figure 3

Simulation of steps in Reaction Systems in case of mutation in $\beta$-globin gene 


\subsection{Validation of Current Strategies to Induce $\gamma$-globin Gene Expression}

\subsubsection{HDAC1/2 Inhibition}

Drugs such as Lovastatin [11], Romidepsin, and Vorinostat [5] can be used as inhibitors of Histone deacetylase enzyme. By eliminating " $\mathrm{H}$ " from initial state, we can simulate this case. Initial state is $\{C, G, F, S, \gamma, M\}$. Then reactions indexed by $1,3,8,10,12,13,14,15,16$ and 19 are enabled. After first round, new state is $\{C, K, G, F, S, S F G, \gamma, M\}$. Then reactions $1,2,3,8,10,13,14,15$, 16 , and 19 are allowed. After second round, the new state $\{C, K, B, G, F, S, S F G$, $\gamma, M\}$ is obtained. Then reactions with numbers $1,2,3,10,13,14,15,16$ and 19 are allowed, which leads to the same state that is the fix point of the system and does not change anymore. Thus HDAC1/2 inhibition has resulted in $\gamma$-globin gene expression induction. The simulation steps are shown in Fig. 4. The simulation works well in this case too.

$$
\{C, G, F, S, \gamma, M\}
$$

Where the allowed reactions have the following indices:

$1,3,8,10,12,13,14,15,16,19$

$\{C, K, G, F, S, S F G, \gamma, M\}$

Where the allowed reactions have the following indices:

$1,2,3,8,10,13,14,15,16,19$

$\{C, K, B, G, F, S, S F G, \gamma, M\}$

Where the allowed reactions have the following indices:

$1,2,3,10,13,14,15,16,19$

$\{C, K, B, G, F, S, S F G, \gamma, M\}$

Figure 4

Simulation of steps in Reaction Systems in case of HDAC1/2 inhibition

\subsubsection{KLF1 Down-Regulation}

A recent example of KLF1 down regulator is herbal drug Ninjin'yoeito [10]. To simulate it, we have considered initial state to be: $\{C, D, H, G, F, S, \gamma, M\}$. Then reaction numbers $3,4,8,10,12,13,14,15,16,17$ and 19 are allowed. After the 
first round, the state $\{C, D, H, N, G, F, S, S F G, \gamma, M\}$ is reached. Then reactions 3, $4,8,13,14,15,16,17$ and 19 are allowed. The last state, which is the fix point of the system and does not change anymore is the same as previous one $\{C, D, H, N$, $G, F, S, S F G, \gamma, M\}$, which means down regulation of KLF1 has resulted in $\gamma$ globin gene expression induction. Down-regulation of KLF1 leads to down regulation of $\beta$-globin gene, which results in the induction of $\gamma$-globin gene expression. Thus, this scenario might be useful only in the case of severe $\beta$ thalassemia cases, where the production of $\beta$-globin gene is already defective or no $\beta$-globin is expressed at all. The stages are explained in Fig. 5.

$\{C, D, H, G, F, S, \gamma, M\}$

Where the allowed reactions have the following indices:
$3,4,8,10,12,13,14,15,16,17,19$
$H, N, G, F, S, S F G, \gamma, M\}$
Where the allowed reactions have the following indices:
$3,4,8,13,14,15,16,17,19$
$\begin{aligned} & 3, N, G, F, S, S F G, \gamma, M\}\end{aligned}$

Figure 5

Simulation of steps in Reaction Systems in case of KLF1 down-regulation

\subsubsection{BCL11A Down-Regulation}

Hydroxyurea (HU) [9] and simvastatin together with t-BHQ decrease expression of BCL11A gene [13]. To simulate it, we have defined object $I$ to represent inhibition of BCL11A. To simulate such case, we considered initial state to be $\{C$, $H, G, F, S, \gamma, M, I\}$. Then reactions $1,3,4,8,10,12,13,14,15,16,18$ and 19 are allowed. New state after first round is $\{C, K, H, N, G, F, S, S F G, \gamma, M, I\}$. Then reactions $1,3,4,8,13,14,15,16,18$ and 19 are enabled. The last state which is the fix point of the system which does not change anymore is $\{C, K, H, N, G, F, S$, $S F G, \gamma, M, I\}$. Thus, the simulation shows that down regulation of BCL11A leads to $\gamma$-globin gene expression induction. The simulation stages are illustrated in Figure 6. Our model also captures well that type of treatment. 
$\{C, H, G, F, S, \gamma, M, I\}$

\section{Where the allowed reactions have the following indices:}

$1,3,4,8,10,12,13,14,15,16,18,19$

$\{C, K, H, N, G, F, S, S F G, \gamma, M, I\}$

Where the allowed reactions have the following indices:

$1,3,4,8,13,14,15,16,18,19$

$\{C, K, H, N, G, F, S, S F G, \gamma, M, I\}$

Figure 6

Simulation of steps in Reaction Systems in case of BCL11A down-regulation

\subsubsection{SOX6 Down-Regulation}

There are drugs such as HU which their utilization leads to SOX6 down regulation [9]. To simulate, we may consider initial state as: $\{C, H, G, F, \gamma, M\}$. Then reactions indexed by numbers $1,4,8,10,12,13,14,15$, and 16 are allowed. After the first round, new state is $\{C, K, H, N, G, F, \gamma, M\}$. Then reactions $1,2,4,8,12$, $13,14,15$, and 16 are allowed. After second round, new state is $\{C, K, B, H, N, G$, $F, \gamma, M\}$. Then reactions indexed by $1,2,4,12,13,14,15$, and 16 are allowed. After third round, new state is the same as previous one, which is the fix point of the system and does not change anymore. Thus, down regulation of SOX6 led to $\gamma$-globin gene induction. The simulation steps are shown in Fig. 7. The model gives the expected result.

\subsubsection{GATA1 Inhibition}

GATA1 can reverse $\gamma$-globin gene silencing [19]. To simulate, we may consider initial state as: $\{C, H, S, F, \gamma, M\}$. Then reactions numbered by $1,4,8,10,12,14$, 15,16 and 19 are allowed. After first round, the new state is $\{C, K, H, N, S, F, \gamma$, $M\}$. Then reactions indexed by $1,2,4,8,12,14,15,16$ and 19 are allowed. After second round, new state is $\{C, K, B, H, N, S, F, \gamma, M\}$. Then reactions $1,2,4,12$, $14,15,16$ and 19 are allowed. After third round, new state is the same previous which is the fix point of the system and does not change anymore. Therefore, GATA1 inhibition leads to $\gamma$-globin gene expression induction. The simulation steps are demonstrated in Fig. 8. 


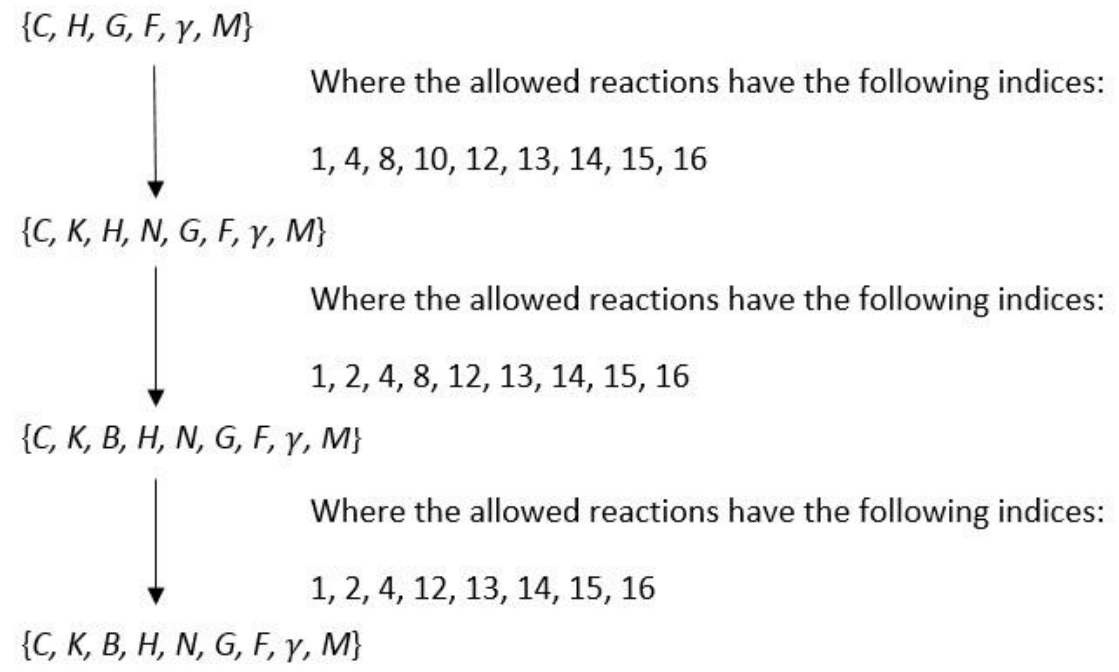

Where the allowed reactions have the following indices: $1,2,4,8,12,13,14,15,16$

$\{C, K, B, H, N, G, F, \gamma, M\}$

Where the allowed reactions have the following indices: $1,2,4,12,13,14,15,16$

$\{C, K, B, H, N, G, F, \gamma, M\}$

Figure 7

Simulation of steps in Reaction Systems in case of SOX6 down-regulation

$\{C, H, S, F, \gamma, M\}$
$\{C, K, H, N, S, F, \gamma, M\}$
$\{C, K, B, H, N, S, F, \gamma, M\}$
$\{C, K, B, H, N, S, F, \gamma, M\}$

Figure 8

Simulation of steps in Reaction Systems in case of GATA1 inhibition

\subsection{Predicting Result of a Novel Strategy in Favor of Gamma- globin Gene Expression Induction}

In this subsection we identify a new possibility for treatment of $\beta$-thalassemia based on our model. 


\subsubsection{RNA Interference (RNAi)}

A powerful methodology for studying the function of any gene is to experimentally disrupt its expression to examine the resulting phenotype. RNA interference (RNAi) is a naturally occurring mechanism of gene regulation. This can be induced by the introduction of double stranded RNA into a cell. This event can be synthetically utilized to down-regulate expression of specific genes by transfecting mammalian cells with synthetic short interfering RNAs (siRNAs). These siRNAs can be designed to silence the expression of genes of interest having a certain target sequence, and may potentially be presented as a therapeutic strategy for inhibiting transcriptional regulation of genes [15].

\subsubsection{Inhibition of FOG1 by RNAi Methodology}

To simulate, we may consider initial state as: $\{C, H, G, S, \gamma, M\}$. Then reactions indexed by $1,4,8,10,12,13,15,16$ and 19 are allowed. After first round, new state is $\{\mathrm{C}, \mathrm{K}, \mathrm{H}, \mathrm{N}, \mathrm{G}, \mathrm{S}, \gamma, \mathrm{M}\}$. In this state the reactions indexed by $1,2,4,8$, $12,13,15,16$ and 19 are allowed. After second round, the new state is $\{C, K, B$, $H, N, G, S, \gamma, M\}$. Then reactions numbered by $1,2,4,12,13,15,16$ and 19 are allowed. After third round, the new state is the same as previous one, which is the fix point of the system and does not change anymore. Therefore, FOG1 inhibition has led to $\gamma$-globin gene expression induction as a possible. The steps of this simulation process are illustrated in Fig. 9.

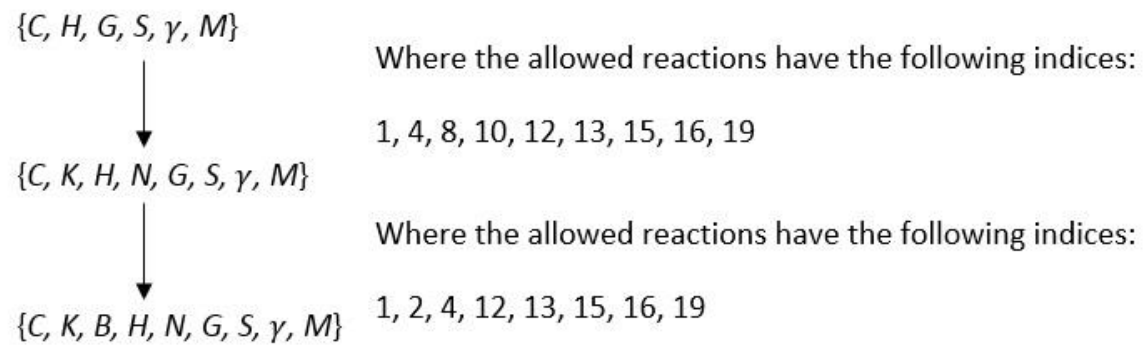

Figure 9

Simulation of steps in Reaction Systems in case of FOG1 inhibition by RNAi

\section{Discussion}

In this section, we give a brief comparison of our simulation model and other models. Various simulation techniques were used for $\beta$-thalassemia, e.g., in [1, 16]. However, in these papers the authors only wanted to simulate and understand 
the disease itself, simulating how the gene can produce hemoglobin, and what is wrong in case of $\beta$-thalassemia, without proposing or checking any possible treatments.

The nature of the considered problem results relatively simple simulations, after a few steps the system halts in each case. The same phenomenon occurs by simulation with Petri nets [14], the simulation goes without any cycles. In [14] hybrid places (both discrete and continuous) were used instead of only discrete ones. Therefore, instead of a discrete value (integer number of tokens), real numbers were used at each place. In this way, in the simulation Petri times were used, e.g., the simulation program ran for 500 Petri times. The simulation results of Subsections 5.2.1, 5.2.2, 5.2.3 and 5.2.4 are in line with the simulation results with the Hybrid Functional Petri nets model, where HDAC1/2 gene expression was inhibited using ST-20 drug, KLF-1 gene expression was inhibited using MS275, ST-20, and the combination of Simvastatin and tBHQ drugs, BCL11A gene expression was inhibited using ACY-957 drug and SOX6 gene expression was inhibited using ACY-957 drug, respectively [14]. Our reaction systems model had the advantage to simplify all this. We could answer whether a treatment can be useful or not in just a few steps. The advantage of the binary feature of the reaction systems approach is that it simplifies the system analysis. Such method can be used as a pre-test to identify potential entities which can play an important role to up-regulate or down-regulate a specific component in the system. After identifying the entities which play an important role, it will be possible to extend the model into a model which is appropriate to investigate the role of these components more accurately (in terms of quantities, ratios, concentrations etc.). The disadvantage of our binary approach could be that it cannot investigate the role of each entity in the system quantitatively. Although, this approach shows which manipulation of the system can be useful to up-regulate or down-regulate the target entity, it does not provide any sort of comparison between the efficiency of the possible treatments. To compare the treatments quantitatively, hybrid models can be used such as hybrid functional Petri nets [14] and fuzzy stochastic hybrid Petri nets [12]. We underline that the proposed strategies shown in Subsections 5.2.5 and 5.3 are novel and up to our knowledge they have not been simulated with other methods, nor biological experiments are done to verify them.

Each of the known strategies proposed for treatment of $\beta$-thalassemia we have checked with simulations were in line with lab experiments. This model shows also a nice example, how the analysis (through simulation) of biological systems such as hemoglobin switching network can be useful. This approach can be used as a pre-test to analyze more complex biological systems in future studies.

\section{Conclusions}

We have demonstrated that "Reaction Systems" is a beneficial model, that simulates pathways, such as, fetal to adult hemoglobin switching developmental stage, in healthy people, thalassemic or sickle cell anemia patients and in case of 
various treatments to compensate for the lack of $\beta$-globin gene expression via $\gamma$ globin gene expression induction by down-regulation or inhibition of gene expressions such as HDAC1/2, KLF1, BCL11A, SOX6, and GATA1. The simulation results in our Reaction Systems model shows that $\beta$-globin gene expression is upregulated as expected in adult stage, and downregulated in patients who are suffering from $\beta$-globin gene related disorders caused by mutation in $\beta$-globin gene. Moreover, simulation results of our proposed model demonstrate that inhibition of HDAC1/2 gene expression decreases the concentration level of NuRD complex, down-regulation of KLF1 gene expression decreases gene expression of BCL11A, down-regulation of BCL11A gene expression decreases the binding rates of $\gamma$-globin gene and the multi-protein complex including BCL11A, NuRD, and erythroid transcription factors (GATA1, FOG1, and SOX6), and finally down-regulation of SOX6 gene expression and inhibition of gene expression of GATA1 decreases the concentration of erythroid transcription factors. In all cases, $\gamma$-globin gene up-regulated in adult stage, which agree with current known treatments of $\beta$-globin gene related disorders via $\gamma$ globin gene expression induction. Therefore, since hemoglobin switching process can be represented as binary of gene expression up regulation or down regulation, our proposed reaction systems model can describe and analyze this biological phenomenon.

Moreover, we propose a novel strategy to treat $\beta$-thalassemia and sickle cell anemia by inhibiting expression of FOG1 by using methods such as RNAi. RNAi as a naturally occurring mechanism of gene regulation method can be used to inhibit FOG1 gene expression by introducing double stranded RNA into the cell. As the results of our simulation have illustrated, this strategy decreases concentration level of erythroid transcription factors and leads to $\gamma$-globin gene expression induction, that is, this proposed strategy could provide potential treatment options. However, we have not performed any laboratory experiments to explore how the proposed strategy would work in a wet lab setting. Thus, further validation of this strategy needs to be performed in our future studies.

\section{Acknowledgement}

The authors would like to acknowledge Prof. Dr. Rza Bashirov for his continuous support and encouragement in our scientific endeavors.

\section{References}

[1] AbdulAzeez S, Borgio JF (2016) In-silico computing of the most deleterious nsSNPs in HBA1 gene. PloS one 11(1), e0147702

[2] Bank A (2006) Regulation of human fetal hemoglobin: new players, new complexities. Blood 107(2):435-43

[3] Bauer DE, Kamran SC, Orkin SH (2012) Reawakening fetal hemoglobin: Prospects for new therapies for the Beta-globin disorders. Blood 120(15):2945-53 
[4] Breton A, Theodorou A, Aktuna S et al (2016) ASH1L (a histone methyltransferase protein) is a novel candidate globin gene regulator revealed by genetic study of an English family with beta-thalassaemia unlinked to the beta-globin locus. British journal of haematology 175(3):525-530

[5] Cao DJ, Wang ZV, Battiprolu PL et al (2011) Histone deacetylase (HDAC) inhibitors attenuate cardiac hypertrophy by suppressing autophagy. Proc Natl Acad Sci U S A 108(10):4123-8

[6] Ehrenfeucht A, Rozenberg G (2007) Events and modules in reaction systems. Theoretical Computer Science 376(1-2):3-16

[7] Ehrenfeucht A, Rozenberg G (2004) Basic Notations of Reaction Systems, Developments in Language Theory. LNCS 3340:27-29

[8] Galanello R, Origa R (2010) Beta-thalassemia. Orphanet J Rare Dis 5(11)

[9] Grieco AJ, Billett HH, Green NS et al (2015) Variation in Gamma-Globin Expression before and after Induction with Hydroxyurea Associated with BCL11A, KLF1 and TAL1. PLoS, One 10(6):e0129431

[10] Inoue T, Kulkeaw K, Muennu K et al (2015) Herbal drug ninjin'yoeito accelerates myelopoiesis but not erythropoiesis in vitro. Genes Cells 19(5):432-40

[11] Lin YC, Lin JH, Chou CW et al (2008) Statins increase P21 through inhibition of Histone Deacetylase Activity and release of promoterassociated HDAC1/2. American Association for Cancer Research 68(7):2378-83

[12] Liu F, Heiner M, Yang M (2016) Fuzzy stochastic petri nets for modeling biological systems with uncertain kinetic parameters. PloS one 11(2), e0149674

[13] Macari ER, Shaeffer EK, West RJ, Lowrey CH (2013) Simvastatin and tbutylhydroquinone suppress KLF1 and BCL11A gene expression and additively increase fetal hemoglobin in primary human erythroid cells. Blood 121(5):830-9

[14] Mehraei M, Bashirov R, Tüzmen Ş (2016) Target-based drug discovery for $\beta$-globin disorders: drug target prediction using quantitative modeling with hybrid functional Petri nets. Journal of bioinformatics and computational biology 14(05), 1650026

[15] Ozcan G, Ozpolat B, Coleman R et al (2015) Preclinical and clinical development of siRNA-based therapeutics. Advanced drug delivery reviews 87:108-119

[16] Paokanta P, Harnpornchai N, Srichairatanakool S et al (2011) The Knowledge Discovery of $\beta$-Thalassemia Using Principal Components 
Analysis: PCA and Machine Learning Techniques. International Journal of e-Education, e-Business, e-Management and e-Learning, 1(2), 169

[17] Sankaran VG (2011) Targeted Therapeutic Strategies for Fetal Hemoglobin Induction. Hematology Am Soc Hematol Educ Program 1:459-65

[18] Suzuki M, Yamamoto M, Engel JD (2014) Fetal globin gene repressors as drug targets for molecular therapies to treat $\beta$-globinopathies. Mol Cell Biol 34(19):3560-9

[19] Yao X, Kodeboyina S, Liu L et al (2009) Role of Stat3 and GATA-1 Interactions in $\gamma$-Globin Gene Expression. Exp Hematol 37(8):889-900 